\title{
Insulin action on metabolism
}

\author{
K J . H eesom ${ }^{2}$, M . H arbeck ${ }^{1}$, C.R . K ahn ${ }^{3}$, R . M. D enton ${ }^{2}$ \\ ${ }^{1}$ Jefferson Medical College, Philadelphia, Pennsylvania, USA \\ ${ }^{2}$ University of Bristol Department of Biochemistry, School of Medical Sciences, Bristol, UK \\ ${ }^{3}$ Joslin Diabetes Center, Boston, Massachusetts, USA
}

The metabolic actions of insulin are largely on muscle, liver and adipose tissue. Insulin increases the uptake of glucose from the blood and enhances its conversion to glycogen and triglyceride. The hormone also promotes the synthesis of protein. At the same time insulin can inhibit the breakdown of triglyceride and glycogen - and in the liver can inhibit gluconeogenesis and ketogenesis. These actions are brought about by a combination of rapid effects (such as the stimulation of glucose transport in fat and muscle cells and the alteration of the activity of key enzymes in metabolism by changes in their phosphorylation) and more long term mechanisms which involve changes in gene expression by the regulation of DNA transcription and changes in the rate of translation.

Since the first JDFI conference only 12 years ago, tremendous progress has been made in elucidating the mechanisms of insulin action at the molecular level. In 1985 essentially none of the intracellular components which make up the signal transduction system which links the insulin receptor to the many intracellular effects of insulin were known. Now, many components are well recognized. Altogether, the insulin signal transduction system is remarkably complex involving the initial tyrosine phosphorylation of

Participants: M. White, Joslin Diabetes Center Research Division, Boston, Massachusetts, USA

J.C. Lawrence, Jr., Department of Pharmacology, University of Virginia School of Medicine, Charlottesville, Virginia, USA

B. Goldstein, Thomas Jefferson University, Philadelphia, Pennsylvania, USA

M. Czech, University of Massachusetts Medical Center, Worcester, Massachusetts, USA

Corresponding author: Professor R.M. Denton, University of Bristol, Department of Biochemistry, School of Medical Sciences, University Walk, Bristol, BS8 1TD, UK a number of intracellular proteins. This gives rise to the activation of Ras, phosphatidylinositol-3 kinase and other elements and hence to the activation of a number of serine/threonine protein kinases - many of which are arranged in cascades. Altogether a truly spectacular increase in knowledge. Moreover, this knowledge has been very important in increasing understanding of intracellular signalling in other areas including the signal transduction pathways involved in the action of growth factors (e.g. insulin like growth factor (IGF)-1 and nerve growth factor), cytokines and beta-cell growth and differentiation.

The talks in this session reflected progress in five major areas within the field of insulin action.

Morris White: The insulin receptor substrate (IRS) signalling system: a crossroad for insulin and cytokine action

Tyrosine phosphorylation of "docking proteins" by the insulin receptor plays a key role in insulin signalling. These docking proteins provide a common interface between various signalling proteins with Src-homology-2 domains ( $\mathrm{SH} 2$ proteins) and the receptors for insulin, IGF-1 and other cytokines. The insulin receptor substrate IRS-1 was the first docking protein identified and serves as a prototype for this class of molecule.

Morris White summarized the structure and role of IRS-1 and other docking proteins which act as insulin receptor substrates such as IRS-2, Gab-1 and p62 ${ }^{\text {doc }}$. These proteins are not related by extensive amino acid sequence identity, but are related functionally as insulin receptor substrates. IRS proteins are recognized by their common functional features, including an $\mathrm{NH}^{2}$-terminal pleckstrin homology $(\mathrm{PH})$ domain and/or phosphotyrosine binding PTB domain which mediate protein-lipid or protein-protein interactions; 
multiple tyrosine residues which form $\mathrm{SH} 2$ binding sites; proline-rich motifs to engage $\mathrm{SH} 3$ motifs or WW domains; and serine/threonine rich regions which may regulate overall function through other protein-protein interactions or phosphorylation events. Other docking proteins have been identified including Shc, p130 cas and sin. Shc is composed of an $\mathrm{NH}^{2}$-terminal PTB domain, a $\mathrm{COOH}$-terminal $\mathrm{SH} 2$ domain, an intervening proline-rich motif, and a few tyrosine phosphorylation sites, one of which engages the SH2 domain of Grb-2. The PTB and SH2 domains provide Shc with considerable promiscuity in its interplay with activated receptors, but without a $\mathrm{PH}$ domain commonly found in IRS proteins, Shc is a relatively weak insulin receptor substrate. The p130 ${ }^{\text {cas }}$ and sin proteins posses SH3 domains which couple these docking proteins to Src kinase but precludes their interaction with the insulin receptor. The future holds considerable promise for the discovery of new docking proteins to coordinate signalling from the insulin and other receptors.

What signalling advantages do the IRS proteins provide? Firstly, IRS proteins provide a mechanism for signal amplification by eliminating the stoichiometric restraints encountered by receptors which directly recruit $\mathrm{SH} 2$ proteins to their autophosphorylation sites. Secondly, IRS proteins may dissociate the intracellular signalling complex from the endocytic pathways of the activated receptor. Thirdly, the interaction of a single insulin receptor with multiple IRS protein isoforms expands the repertoire of signalling pathways that can be regulated. Finally, the use of shared IRS proteins by multiple receptors provides a mechanism to integrate several receptor systems through a single complex.

The major mechanism which generates signals by activated IRS-proteins is the binding of $\mathrm{SH} 2$ proteins to tyrosine phosphorylated proteins including phosphatidylinositol-3 kinase (p85 $\left.\alpha / \mathrm{p} 55 \alpha, \mathrm{p} 85 \beta, \mathrm{p} 55^{\mathrm{pik}}\right)$, Grb-2, SHP-2, fyn, nck and crk. The phosphatidylinositol-3 kinase and SHP-2 are enzymes that are activated during association with IRS-1; nck, crk and Grb-2 are adapter molecules which link activated IRS proteins to various signalling pathways including Ras. In addition however, IRS proteins may mediate additional signals which depend upon serine phosphorylation or other protein-protein interactions.

In parallel with the work described above, a large group of cytokines and growth factors also engage and phosphorylate IRS proteins, including IGF-1 and growth hormone, members of the interleukin (IL)-2 family of cytokines (IL-2, IL-4, IL-9, IL-13 and IL-15), IL-6 related cytokines (oncostarin M. OSM, leukocyte inhibitory factor LIF and leptin) and interferons. This crosstalk raises the possibility that disruption of insulin signalling and the subsequent alteration of other signalling systems may contribute to disease.
Richard M. Denton: The role of serine/threonine protein kinases in insulin action

The activation of the intracellular tyrosine kinase activity of the insulin receptor leads to autophosphorylation of the receptor as well as the phosphorylation of a number of intracellular proteins. This, in turn, gives rise to the activation of Ras and phosphatidylinositol 3-kinase and hence to the activation of a number of serine/threonine protein kinases. Many of these kinases appear to be arranged in cascades. A full understanding of the nature of these cascades and the cross-talk between them is crucial to understanding the metabolic effects of insulin within the cell and how these effects may be altered in the disease state.

Richard Denton discussed the role of various protein kinase cascades in the stimulation of glycogen, fatty acid and protein synthesis by insulin in rat epididymal fat cells. These cells remain an important model system for studying the signalling pathways in insulin action. Effects of insulin on glucose transport, fatty acid, glycogen and protein synthesis and on lipolysis can all be studied in the same cell. The cells are fully differentiated, non-dividing and reflect the actual effects of insulin within the intact animal. In contrast, the effects of insulin on many stable cell lines are apparently related to cell growth and division and effects on metabolism are often modest. It is useful to compare the metabolic effects of insulin with those of epidermal growth factor, since these two agents have contrasting effects on two distinct kinase cascades. The cascade resulting in the activation of MAP kinase is stimulated to a greater extent by epidermal growth factor than by insulin, whereas the opposite is true for the cascade leading to the activation of p70 S6 kinase. The effects of wortmannin, which inhibits phosphatidylinositol 3-kinase, and of rapamycin, which blocks the activation of p70 S6 kinase, are also useful tools for exploring the roles of these components in the specific actions of insulin.

Such approaches have indicated that the mitogen activated protein (MAP)-kinase cascade is probably not important in the acute metabolic effects of insulin, but may play a role in the regulation of gene transcription and hence in the more long term effects of insulin. The short term metabolic effects of insulin appear to involve at least three distinct signalling pathways: (i) those leading to increases in glucose transport and the activation of glycogen synthase (and hence glycogen synthesis), acetyl-CoA carboxylase (and hence fatty acid synthesis), eIF-2B (and protein synthesis) and phosphodiesterase (stimulating lipolysis) which may involve phosphatidylinositol 3-kinase and possibly protein kinase B, (ii) those leading to some of the effects of insulin on protein synthesis (including formation of eIF-4F complex, ribosomal protein $\mathrm{S} 6$ phosphorylation and activation 
of eEF-2) which may involve phosphatidylinositol 3kinase and p70 S6 kinase, and finally, (iii) those leading to the activation of pyruvate dehydrogenase (and the stimulation of fatty acid synthesis) which is apparently unique among the metabolic effects of insulin in not requiring phosphatidylinositol 3-kinase.

Strategies are currently being developed to extend the range of techniques which can be applied to rat fat cells. For example, Richard Denton described how these cells can be made permeable to small molecules by electroporation, which allows entry of small molecules which otherwise would be excluded while signalling remains relatively intact. In this way, a wide range of specific inhibitors and other agents can be introduced into cells, and the resulting alterations in the metabolic effects of insulin studied. Electroporation of these cells also allows remarkably high levels of transfection with a variety of plasmids, again allowing manipulation of known and putative components of the insulin signalling system. For example, after transfection with plasmid constructs expressing tagged protein kinase $\mathrm{B}$, both the endogenous protein kinase $\mathrm{B}$ and the expressed protein kinase B can be activated by insulin. Present studies are using this system to explore the upstream events which result in the activation of protein kinase $B$, as well as the role of this kinase in regulating the metabolic effects of insulin.

The use of systems to follow insulin action in single cells would appear to offer further powerful approaches to identify components involved in insulin signal transduction. These systems allow the effects of insulin to be followed in real time and a wide range of potential reagents which intervene in the signalling pathways can be introduced into cells by microinjection. In addition, this approach only requires a tiny sample of original tissue, which is of particular importance when the techniques are applied to cells of human origin where changes associated with human diabetes can be explored directly. Two examples developed in Bristol by Jeremy Tavaré and Guy Rutter were described. In the first, the use of an ultrasensitive charge coupled device (CCD) camera allows the expression of luciferase in single living cells to be followed and in this way the signalling pathways involved in the regulation of a range of promoters controlled by insulin can be studied. In the second, expression of chimeras of the glucose transporter GLUT-4 and the highly fluorescent green fluorescent protein (GFP) allow the translocation of GLUT-4 to the plasma membrane to be followed in real time in living cells.

John C. Lawrence, Jr.: Insulin action on protein synthesis

The stimulation of protein synthesis by insulin occurs in a wide variety of cell types and can happen within minutes of a rise in the concentration of this hormone. In many cell types, the initiation phase is rate limiting for mRNA translation and is an important site of regulation. Initiation involves recognition of the mRNA by eIF-4E, the mRNA cap-binding protein, which together with eIF-4A and eIF-4G form the eIF-4F initiation complex.

John C. Lawrence, Jr. discussed the way that the formation of this complex is regulated by insulin. PHAS-1 (also referred to as 4E-BP1) is a phosphorylated heat- and acid-stable protein first detected around 1980 as one of several species that were phosphorylated when rat adipocytes were incubated with insulin. PHAS-1 is now known to be the first member of a family of proteins that mediate the effects of insulin and growth factors on mRNA translation. Nonphosphorylated PHAS-1 binds tightly to eIF-4E and prevents it from binding to eIF-4G. Thus, PHAS-1 blocks formation of the initiation complex that is essential for efficient binding and/or scanning of the mRNA by the 40S ribosomal subunit. When PHAS1 is phosphorylated in response to insulin or growth factors it dissociates from 4E, thereby allowing $4 \mathrm{E}$ to participate in translation initiation. This effect explains, in part, the stimulation of the overall rate of protein synthesis in response to insulin. It also explains the selective expression of messages that contain secondary structure in their $5^{\prime}$ untranslated region (5'UTR), which are particularly dependent on eIF-4E for translation. Interestingly, cAMP, polio virus and encephalomyocarditis (EMC) virus promote dephosphorylation of PHAS-1 and increase the binding of this protein to eIF-4E, thereby inhibiting protein synthesis.

Early studies suggested a possible role for MAP kinase in the regulation of PHAS-1 by insulin, since MAP kinase rapidly phosphorylated recombinant PHAS-1 in vitro. Support for a role of MAP kinase in phosphorylating PHAS-I was provided by the finding that the compound PD098059 promoted dephosphorylation of PHAS-I in chinese hamster ovary (CHO) cells overexpressing the human insulin receptor. PD098059 is a non-competitive inhibitor of MEK, the enzyme that activates MAP kinase. However, blocking activation of MAP kinase in adipocytes with this inhibitor did not attenuate the effects of insulin on this protein, suggesting that the effect of insulin on PHAS-1 in adipocytes is mediated by a MAP kinase-independent pathway.

Although MAP kinase is not the major PHAS-1 kinase in adipocytes, phosphorylation of PHAS-1 by MAP kinase in vitro does decrease the binding of this protein to eIF-4E. Thus, MAP kinase can be used as a tool to identify phosphorylation sites important in the regulation of PHAS-1 in response to insulin. To identify such sites, PHAS-1 was phosphorylated with MAP kinase and $\left[\gamma_{-}{ }^{32} \mathrm{P}\right] \mathrm{ATP}$, then cleaved proteolytically and the resulting phosphopeptides 
isolated by reverse phase chromatography and identified by amino acid sequencing. Phosphorylated residues were located by determining the cycles in which ${ }^{32} \mathrm{P}$ was released when phosphopeptides were subjected to sequential Edman degradation. With an extended incubation in vitro, MAP kinase phosphorylated $\mathrm{Thr}^{36}, \mathrm{Thr}^{45}, \mathrm{Ser}^{64}, \mathrm{Thr}^{69}$ and $\mathrm{Ser}^{82}$. In rat adipocytes the phosphorylation of all five sites was increased in response to insulin and decreased by rapamycin, an inhibitor of the function of mTOR, although there were differences in the magnitude of the effects. A form of PHAS-1 phosphorylated exclusively on $\mathrm{Thr}^{36}$ remained bound to eIF-4E, indicating that phosphorylation of this residue is insufficient for dissociation of the PHAS-1/eIF-4E complex. These results indicate that multiple phosphorylation sites are involved in the control of PHAS-1. All five sites identified fit a (Ser/Thr)-Pro motif, suggesting that the phosphorylation of PHAS-1 in cells in response to insulin may be mediated by a proline-directed kinase other than MAP kinase.

As mentioned above, the effects of insulin on PHAS-1 in adipocytes and other cell types are antagonized by rapamycin and wortmannin, two agents that inhibit the mTOR signalling pathway. In addition, the effects of insulin on PHAS-1 are attenuated by agents that increase intracellular cAMP and also by cAMP derivatives and phosphodiesterase inhibitors. These agents also inhibit the activation of p70S6 kinase, a downstream element of the mTOR pathway. Thus, elements of this pathway appear to be involved in controlling the phosphorylation of PHAS1 by insulin. Indeed, immunoprecipitates of mTOR phosphorylate all five sites described above and this phosphorylation inhibits PHAS-1 binding to eIF-4E, suggesting that mTOR itself, or a kinase tightly associated with it, may contribute to the phosphorylation of PHAS- 1 in cells.

Future studies will aim to determine the exact mechanism by which insulin and growth factors signal to PHAS-1 and the identity of the kinase(s) involved in this pathway. Insulin has other effects on components involved in translation such as the phosphorylation of the ribosomal protein S6, and the regulation of the activities of eIF-2B and eEF-2. Much remains to be elucidated concerning the specific physiological roles of these multiple actions of insulin on protein synthesis.

\section{Michael Czech: The molecular basis of insulin action on glucose transport}

The translocation of insulin-regulated GLUT-4 glucose transporters from intracellular vesicles to the plasma membrane is a key response of insulin sensitive cells to the hormone insulin and is crucial for the maintenance of normal blood glucose levels. The mechanism by which such extracellular stimuli regulate these membrane trafficking processes remains undefined.

Michael Czech described how the activation of phosphatidylinositol (PI) 3-kinase, which phosphorylates the $3^{\prime}$ position of phosphoinositides, may be a central element in the signal transduction pathway involved in this action of insulin. In fact, signalling by 3phosphoinositides may be involved in the regulation of diverse cellular responses, such as protein sorting, cell adhesion and chemotaxis. The identification of several protein targets of 3-phosphoinositides suggests that these phosphoinositides might serve as membrane localization elements, recruiting target proteins to specific cellular organelles. Michael Czech then presented his recent findings on the identification of a family of proteins linking PI 3-kinase signalling pathways to proteins which mediate membrane trafficking.

To elucidate the mechanism by which 3-phosphoinositides mediate extracellular signalling processes, it is necessary to identify effectors of phosphoinositides (i.e. PI binding proteins). A cloning protocol was developed utilizing ${ }^{32} \mathrm{P}$-labelled bovine brain phospholipids, generated by incubation with glutathione S transferase (GST)-p110 (PI 3-K catalytic subunit) fusion protein and $\left[{ }^{32} \mathrm{P}\right] \mathrm{ATP}$, to probe a cDNA library for general receptors for phosphoinositides (GRPs). A single cDNA clone was isolated from both brain and adipocyte expression libraries which encoded a protein termed GRP1. This protein bound $\left[{ }^{32} \mathrm{P}\right] \mathrm{PtdIns}(3,4,5) \mathrm{P}_{3}$, but not $\left[{ }^{32} \mathrm{P}\right] \operatorname{PtdIns}(3,4) \mathrm{P}_{2}$ or $\left[{ }^{32} \mathrm{P}\right] \mathrm{PtdIns}(3) \mathrm{P}$ under the conditions of the library screen. PtdIns $(3,4,5) \mathrm{P}_{3}$ is the product of the phosphorylation at the $3^{\prime}$ position of PtdIns $(4,5) \mathrm{P}_{2}$ by the p110 class of PI 3-kinases, which are regulated by insulin.

GRP1 is similar to the protein cytohesin-1 originally cloned from cytolytic natural killer $\mathrm{T}$ lymphocytes. Both proteins contain pleckstrin homology (PH) domains and the Sec7 homology region. Sec7 is a $230 \mathrm{kDa}$ protein in yeast containing a 200 amino acid domain found in several proteins. Sec7 cycles between cytosolic and membrane vesicles as they pass through the Golgi apparatus. The Sec7 domain of cytohesin-1 enhances cellular adhesion through the direct association with the cytoplasmic region of integrin $\beta 2$. The Sec7 domain of a cytohesin-1 isoform, denoted ARNO (for ARF nucleotide binding site opener), has nucleotide exchange activity for the small guanine nucleotide-binding protein, adenosine diphosphate-ribosylation factor 1 (ARF1). In the presented studies, GRP-1 was shown to exhibit ARF1 exchange activity. Moreover, $\operatorname{PtdIns}(3,4,5) \mathrm{P}_{3}$ was shown to enhance this activity fourfold. In yeast, ARF activation (GTP-GDP exchange) is regulated by Sec7-domain proteins and is involved in vesicle budding and fission. 
GST fusion proteins containing residues 239 to 399 (the PH domain), residues 52 to 260 (the Sec7 domain), residues 5 to 71 , or residues 5 to 399 of GRP1 were used to determine whether $\operatorname{PtdIns}(3,4,5) \mathrm{P}_{3}$ binding could be localized to specific sequences within the GRP1 structure. Nearly full-length GRP1 and the $\mathrm{PH}$ domain bound to $\mathrm{PtdIns}(3,4,5) \mathrm{P}_{3}$ were able to do so, whereas the fusion proteins containing only the Sec7 homology region or the amino terminal sequence did not. The association of GRP1 PH domain (and the cytohesin-1 PH domain) was specific for PtdIns $(3,4,5) \mathrm{P}_{3}$ and did not bind $\operatorname{PtdIns}(3,4) \mathrm{P}_{2}$ or PtdIns(3)P. Binding of PtdIns $(3,4,5) \mathrm{P}_{3}$ to fusion proteins containing the $\mathrm{PH}$ domain of Son of Sevenless (SOS) or IRS-1 were negligible, demonstrating that the preference of the GRP1/cytohesin-1 PH domain for 3-phosphoinositides is not a general property of all $\mathrm{PH}$ domains.

The selectivity of the GRP1 and cytohesin-1 PH domains for PtdIns $(3,4,5) \mathrm{P}_{3}$ suggests that the $\mathrm{PH}$ domain might function in the recruitment of these proteins to sites of $\operatorname{PtdIns}(3,4,5) \mathrm{P}_{3}$ synthesis in response to receptor-regulated activation of PI 3-kinase activity. This mechanism would then define the sites of action of GRP1/cytohesin-1/ARNO proteins, which can in turn regulate the activity of ARF1. This family of proteins might therefore mediate the regulation of protein sorting and membrane trafficking by receptor-activated PI 3-kinase activity.

A number of unresolved issues remain to be addressed before a complete picture of the mechanism linking the activated insulin receptor to GLUT-4 translocation can be constructed. Although GTPbinding proteins of the Rab family have been implicated in the regulation of glucose transport, there is as yet no evidence that ARF1 proteins are involved in GLUT-4 vesicle trafficking. It is also unknown whether PI 3-kinase activity is localized to the specific sites of vesicular trafficking, and if so, by what mechanism does this targeting of PI 3-kinase occur. An increased understanding of these issues might ultimately lead to the development of novel therapeutic agents that can augment glucose transport, thereby enhancing the maintenance of glucose homeostasis.

Barry Goldstein: Regulation of insulin action by protein tyrosine phosphatases

Protein tyrosine phosphatases (PTPases) have recently been shown to play an integral role in the regulation of reversible tyrosine phosphorylation in a number of cellular signalling pathways, including those regulating insulin action. PTPase enzymes regulate the steady-state activity of the insulin receptor kinase by dephosphorylating both the active (autophosphorylated) form of the receptor and phosphotyrosine residues on post-receptor substrates in the insulin signalling pathway, such as IRS-1. Among the variety of PTPases found in insulin sensitive tissues, work from a number of laboratories has provided evidence for a role for the leukocyte common antigen-related (LAR) phosphatase and PTP1B in the negative regulation of insulin action in intact cells, and other candidate PTPases may also play a role in insulin receptor regulation.

Barry Goldstein presented his recent work demonstrating the involvement of specific phosphotyrosylphosphatases in insulin signalling pathways. In order to demonstrate a cellular role for PTP1B, Barry Goldstein and colleagues showed that osmotic loading of rat (KRC)-7 hepatoma cells with affinity-purified antibodies that immunoprecipitate and neutralize the enzymatic activity of recombinant rat PTP1B led to a $42 \%$ increase in insulin stimulated DNA synthesis of PI 3-kinase activity, when compared to control cells loaded with pre-immune IgG. Insulin stimulated receptor kinase activity towards an exogenous peptide substrate was also increased by $57 \%$ in the PTP1B antibody loaded cells, and insulin stimulated receptor autophosphorylation and IRS-1 tyrosine phosphorylation were increased 2.2- and 2.0-fold, respectively. Thus, PTP1B can negatively regulate insulin signalling and acts, at least in part, at the level of the insulin receptor.

Previous work has also shown that reducing or augmenting the abundance of the membrane PTPase, LAR, also leads to enhanced or reduced insulin stimulated receptor autophosphorylation, respectively, in a manner that requires expression of LAR within the plasma membrane of the cell. Furthermore, transfection of a site-directed, catalytically inactive LAR mutant acts as a dominant negative and enhances insulin receptor phosphorylation and kinase activity by a mechanism that appears to involve alterations in the phosphorylation state of specific tyrosine residues in the insulin receptor kinase domain. A functional and physical interaction has been demonstrated between LAR and the insulin receptor in the plasma membrane using chemical crosslinking techniques followed by co-immunoprecipitation. Furthermore, LAR appears to be temporally internalized with the insulin receptor into endosomes in insulin-stimulated rat liver cells and is functionally active in the dephosphorylation of the receptor in this subcellular fraction.

Additional data to support the involvement of LAR in insulin signalling in normal physiology comes from recent experiments performed with a transgenic LAR knock-out mouse model (performed by Barry Goldstein in collaboration with J. Ren, L. J. Sweet, G. Cline, W.C. Skarnes and J. N. Livingston). The LAR knock-out mice had significantly lower fasting levels of insulin, glucose and triglycerides, suggesting a heightened level of insulin sensitivity. The knock-out 
mice had $33 \%$ lower steady-state rates of hepatic glucose production and whole body glucose disposal than the control mice. At the high insulin infusion rate $\left(20 \mathrm{mU} \cdot \mathrm{Kg}^{-1} \cdot \mathrm{min}^{-1}\right)$ in a hyperinsulinaemic clamp, glucose production was completely suppressed in wild-type mice, while it remained close to basal levels in the knock-out mice. Glucose disposal during the clamp was increased by 3.4 -fold over basal in the wildtype, while it increased by only 2.1-fold in the knockout. These interesting results indicate a significant resistance to infused insulin in the liver and skeletal muscle of the knock-out mice. Although the exact site of abnormal regulation of insulin signalling in the LAR knock-out is still under study, these data provide strong evidence for a major role of the LAR PTPase in the regulation of insulin action in normal physiology.

In conclusion, PTPases play an essential role in the activation of the insulin receptor kinase as well as at post-receptor sites in the insulin signalling pathway. From the variety of PTPases found in insulin sensitive tissues, ongoing work from a number of laboratories has provided evidence for a role of LAR and PTP1B in the negative regulation of insulin action in intact cells, where both of these PTPases appear to act, at least in part, at the level of the insulin receptor itself. In the future, other candidate PTPases may also be shown to play a role in the regulation of the insulin receptor. The cellular PTPases that regulate the reversible phosphorylation of IRS-1, IRS-2 and Shc have yet to be identified. Further studies are also necessary in human insulin resistant disease states, including obesity and overt non-insulin-dependent diabetes mellitus (NIDDM), to identify specific PTPases whose abnormal expression or activity might be involved in the pathogenesis of clinical insulin resistance. Ultimately, work in this area will pave the way for the development of agents that can inhibit individual PTPases involved in insulin resistant states, in order to augment insulin signalling.

\section{Summary}

It is clear that research of the past decade has produced tremendous advances in our knowledge of insulin action at the molecular level. No longer can this fundamental component of metabolic regulation be viewed as a simple cascade of intracellular reactions, but rather it is a very complex and intricate network which allows the insulin signal to diverge into multiple metabolic pathways, each of which is highly timed and precisely regulated. It is also important to note that the limited size of this conference only allowed presentation of selected topics, and thus, many equally important areas of insulin action were either not covered or only mentioned in passing. This includes such topics as insulin regulation of glycogen metabolism and gene transcription; interaction between the insulin signalling network and the pathways of regulation by other hormones, growth factors and metabolites; the importance of spatial compartmentalization, kinetics and protein isoforms in the "fine tuning" of these signalling systems; and the very important alterations in insulin and hormonal signalling which occur during development and in various physiological and pathophysiological states, including diabetes. Indeed, this session which focused on insulin action represents only one of the many closely related signalling systems whose understanding is important to the treatment and ultimate cure of diabetes and its complications. For example, similar signalling systems utilizing tyrosine, serine and lipid kinases, as well as IRS-1-like phosphotyrosine docking proteins, $\mathrm{SH} 2$ domain proteins, and various regulatory interactions are at the centre of regulation of lymphocytes and the immune system, beta-cell growth and differentiation, and control of the tissues involved in chronic complications of diabetes such as vascular tissues, nerve and kidney. Furthermore, because of the broad use and similarity of these signalling mechanisms, this is one area of research where advances on one system (e.g. insulin action in muscle, fat or liver) may have important direct application and ramifications in others (e.g. IGF-1 action on kidney and nerve, regulation of the immune response by cytokines and T-cell receptor, and the role of tyrosine phosphorylation or PI3-kinase activation on beta-cell growth and differentiation). Thus, in considering our recommendations for future directions, it is important to consider not only questions which are directly derived from the selected presentations on insulin action at this conference, but even more importantly, the whole area of cellular signalling as it relates to diabetes and its complications.

\section{Future directions and recommendations}

- The complexity of insulin action and the integrated nature of metabolic regulation offer a major challenge to providing optimal control and/or cure for diabetes, since a partial correction of metabolic abnormalities may not prevent all the long term diabetic complications. Therefore, it is essential to define the entire set of metabolic and molecular actions of insulin and to establish therapeutic programmes which normalize them.

- We must define the specific pathways for insulin action for each of the hormone's physiological effects, not only on glucose transport and metabolism (which are obviously of interest), but also on lipid and protein metabolism, regulation of gene expression and other aspects of cellular metabolism.

- We must define the role of kinetics and spatial distribution in insulin signalling which provides 
important fine tuning and hormone specificity to insulin action.

- Many significant components of the insulin signalling network remain undefined and even unanticipated. We must employ existing techniques such as expression cloning, differential display, knowledge of the human genome, etc, as well as develop new techniques, to identify these missing elements and define their roles in insulin action and diabetes.

- It is now clear that the insulin signalling pathway interacts with other pathways initiated by many other hormones and growth factors, such as counter-insulin hormones (catecholamines, glucagon, steroids, etc), vascular active hormones (angiotensin II, endothelin and nitrous oxide) and tissue-specific growth factors (IGF-1, etc). The pathways of insulin action in vivo can also be inhibited, mimicked or modified by the co-ordinated action of many substances, including glucose itself, amino acids, fatty acids and other lipids. These pathways must be defined to optimize the effects of hormones on all tissues.

- Protein and lipid phosphorylation cascades, involving protein-protein interactions and generation of phosphorylated phosphoinositides, are common to a variety of signal transduction systems, indicating the central role these signalling systems play in many cell types related to both insulin-dependent diabetes (IDDM) and NIDDM. These include muscle, fat and liver for insulin action, beta-cells of the pancreas, $\mathrm{T}$ and $\mathrm{B}$ lymphocytes involved in autoimmunity, and vascular and other tissues involved in diabetic complications. Thus, understanding the protein effectors of these secondary messengers is critical to sorting out the underlying mechanisms that may be involved in IDDM and NIDDM and their complications.

- Systems should be developed to accurately reflect the actions of insulin and interacting hormones in the human situation. These systems would include, well-differentiated human cellular systems for muscle, fat, liver and other tissues, transgenic models with metabolic systems which can be regulated at appropriate times in development and which accurately mimic human physiology, as well as cell-free systems in which all the components of a response can be studied in a fully defined milieu.

- Some information regarding insulin and hormone action at the molecular level derived from cellular systems and other models may not reflect the more integrated physiology of normal or diabetic humans. Therefore, we must develop new techniques to evaluate specific metabolic signalling pathways (non-invasively, if possible) in intact human or animal subjects.
- The differences in insulin action in poorly controlled IDDM and NIDDM, as well as other states of insulin resistance, such as obesity and hypertension, need to be established and their impact explored.

- We must establish the extent to which alterations in insulin action play a role in the chronic complications of diabetes in vascular and other non-classical target tissues. This includes studies of interacting hormones and growth factors.

- Rapidly expanding information about the human genome has indicated genetically defined microheterogeneity in insulin signalling. This includes, polymorphisms in IRS-1, PI 3-kinase, glycogen synthase and many other signalling proteins. We must evaluate the impact of this genetic heterogeneity in insulin signalling in terms of its impact on the pathogenesis of both IDDM and NIDDM and also on its impact on diabetes therapeutics. For example, will optimal control at a molecular level be identical in all individuals and ethnic groups, and if not, how can we adjust for these differences?

- A key question is whether information about insulin action can be used to develop substances which mimic or sensitize this action, and if so, whether any of these substances would be superior to insulin itself in the control of diabetes. Investigation of the properties which make a more ideal drug are necessary (for example, orally administered, lower risk of hypoglycaemia, better control in all tissues, etc). Would these drugs be phosphoprotein or phospholipid analogues, or some novel class of molecules?

- Is there a relatively specific phosphatase involved in insulin signalling? If so, can its inhibition enhance insulin action in a therapeutically useful way? Are there changes in the activity or abundance of a specific tyrosine phosphatase which play a role in the abnormal insulin signalling which is common in IDDM and NIDDM and might therefore be a therapeutic target to enhance insulin action?

- Is GLUT4 trafficking directed by lipid products of PI 3-kinase and can these specifically target the trafficking?

In summary, since the first JDFI Conference only 12 years ago, there has been great progress in understanding insulin and other hormone signalling. However, many key questions and formidable challenges remain. Providing answers to these questions and solutions to these challenges will not only impact on our understanding of diabetes, but are essential to optimizing therapy, and ultimately to the cure of the disease. 